\title{
Importance of Hyperkalemia Management in Cardiovascular Disease
}

\author{
0 Korzh* \\ Department of General Practice-Family Medicine, Kharkiv, Ukraine
}

*Corresponding author: 0 Korzh, Department of General Practice-Family Medicine, Kharkiv, Ukraine.

Received Date: August 31, 2019

Published Date: September 06, 2019

\begin{abstract}
Hyperkalemia develops in a patient with systemic arterial hypertension if one or more risk factors are present, namely chronic kidney disease, diabetes mellitus, heart failure, or pharmacological therapy that inhibits potassium homeostasis, mainly by inhibiting renin-angiotensin-aldosterone. Hyperkalemia is a significant cause of morbidity (visits to the emergency department and hospitalization) and portends a higher risk of mortality in patients at risk. There are no clear, specific, standardized guidelines for the treatment of hyperkalemia in patients with chronic kidney disease and/or heart failure, which is problematic given that hyperkalemia can often be a life-threatening condition. The purpose of this review is to identify risk factors for high serum potassium, highlight risk in comparison with the benefits of renin-angiotensin-aldosterone inhibitors for certain patient groups, and describe preventive and therapeutic strategies for hyperkalemia.
\end{abstract}

Keywords: Hyperkalemia; Cardiovascular disease; Kidney disease; Patiromer

\section{Definition, Epidemiology and Risk factors of Hyperkalemia}

Hyperkalemia, which is usually defined as a serum potassium concentration in excess of 5.2 millimoles per liter ( $\mathrm{mmol} / \mathrm{L})$, is a serious illness that, if left untreated, can lead to life-threatening conditions [1,2]. Hyperkalemia can occur as a result of various acute and chronic conditions that affect potassium excretion by the kidneys, and usually occurs in patients with chronic kidney disease (CKD) and other concomitant diseases such as heart failure (HF), diabetes mellitus (DM) and hypertension [3-5]. Hyperkalemia is a serious disease that can cause muscle weakness, paralysis and cardiac arrhythmias, and is associated with increased mortality $[2,5]$. Among people with more than 2 recorded potassium values, from 2007 to 2012 hyperkalemia was detected in $34.6 \%$ of patients with CKD and in $30.0 \%$ of patients with heart failure. In addition, a retrospective study evaluating the 5-year prevalence of hyperkalemia in approximately 1.7 million patients showed that $47.6 \%$ of patients with concomitant CKD from stage 3 to stage 4 and heart failure had at least 1 medical record of hyperkalemia in compared with $8.5 \%$ of patients without CKD or additional concomitant diseases [2,3]. It should be noted that due to the progressive nature of CKD in patients with CKD with or without concomitant diseases, there is a risk of recurrent hyperkalemia. In addition, hyperkalemia can occur without clinical manifestations, which further complicates its diagnosis. With hyperkalemia, cardiac conduction disturbances may occur, as well as violations of depolarization and repolarization. Hyperkalemia can cause the QRS complex to expand, which increases the thresholds of stimulation and can lead to an inability to capture and excessively increase the volume of spaced or spontaneous $\mathrm{T}$ wave using implantable cardioverter defibrillators, which can lead to inappropriate shocks. Finally, hyperkalemia caused electrocardiographic changes (ECG), which were mistaken for malfunctioning pacemakers and implantable defibrillator cardioverter.

ECG changes associated with hyperkalemia can vary between patients, and even normal ECGs have been observed in patients with severe hyperkalemia. Some doctors may look at the ECG and determine that the abnormalities are caused by hyperkalemia, while others do not. This is problematic, as there is no consensus on how to use ECG in the diagnosis and treatment of hyperkalemia. A potential solution to this problem is the development of a diagnostic algorithm that excludes ECG measurements as an accurate indicator of hyperkalemia [6]. The renin-angiotensin-aldosterone system 
(RAAS) plays a critical role in the pathophysiology of CKD and heart failure. Patients are treated with angiotensin-converting enzyme (ACE) inhibitors as the first line of therapy, with angiotensin II receptor blockers (ARBs) used either in combination with ACE inhibitors, or as an alternative to ACE inhibitors in certain patient populations $[7,8]$. Treatment guidelines recommend titration of RAAS inhibitor agents (RAASi) to moderate to high doses so that patients receive optimal treatment benefits. RAASi treatment is critical for treating people with CKD and / or HF; however, these treatments can also be a problem in treating patients with a predisposition to hyperkalemia due to their mechanisms of action, which can increase serum potassium levels. The prevalence of hyperkalemia was generally higher in patients aged 65 years and older than in patients with similar concomitant diseases who were younger than 65 years old. In addition, patients with DM, HF, CKD stage 3-4 and combinations of these conditions were 2.5-5.6 times more likely to experience hyperkalemia compared to the group without any of these conditions. The authors of the study concluded that, with an increase in concomitant medical care, either due to a decrease in renal function, or due to the development of other concomitant conditions, the prevalence of hyperkalemia also increases

Although the frequency and prevalence of hyperkalemia in the general population is not well understood, some studies conducted among hospitalized patients have estimated prevalence from 1 to 10 per 100 patients. In addition, some retrospective analyzes reported that the prevalence of hyperkalemia ranges from $2.5 \%$ to $3.2 \%$ in populations with different risk factors, although the actual prevalence depends on the patient population studied and the serum potassium threshold used to determine hyperkalemia. However, the presence of CKD is constantly associated with hyperkalemia. There are no clear, specific, standardized guidelines for the treatment of hyperkalemia in patients with CKD and / or HF, which is problematic given that hyperkalemia can often be a lifethreatening condition. The treatment approach can vary greatly and may depend on many factors. For example, nephrologists and cardiologists stated that if a patient has a serum potassium level of $5.8 \mathrm{mmol} / \mathrm{L}$, the provider may decide not to treat immediately, but instead wait a few weeks, repeat the test and possibly start treatment based on subsequent laboratory results [9]. Doctors working in emergency rooms indicated that elevated potassium levels usually lead to immediate treatment, followed by referrals to a specific level. If the potassium level is $6 \mathrm{mmol} / \mathrm{L}$ or higher, the patient will receive an emergency referral for nephrology for dialysis; however, if the level is less than $6 \mathrm{mmol} / \mathrm{L}$, patients not suffering from ESRD are usually discharged home for follow-up ambulatory observation with their healthcare provider. Internal medicine doctors may want to control hyperkalemia without referral. Approaches to treatment may depend on the place of medical care, the specialization of the doctor and the level of comfort of the doctor in the treatment of hyperkalemia in patients with initial renal dysfunction and concomitant diseases.
Among the drugs that can lead to or worsen hyperkalemia, RAAS inhibitors make the main contribution. This is problematic because it has been shown that these drugs reduce morbidity and mortality in patients with CKD, DM and cardiovascular disease. RAAS inhibitors, regardless of dose, but especially at higher doses, can cause hyperkalemia, thereby limiting their use in optimal doses for cardiorenal protection. In patients with arterial hypertension who have no risk factors for hyperkalemia, monotherapy with a RAAS inhibitor is associated with a incidence of hyperkalemia of less than $2 \%$, but increases to $5 \%$ when using double RAAS inhibition. The incidence of hyperkalemia is even higher in patients with heart failure or CKD and ranges from 5 to $10 \%[8,9]$.

HF patients are especially susceptible to hyperkalemia caused by a decrease in renal blood flow associated with heart failure, the presence of concomitant diseases such as diabetes, and treatment regimens that include RAAS inhibitors. Patients who received submaximal doses or discontinued RAAS inhibitors had worse cardiorenal results and higher mortality than patients who received maximum doses. The results of these studies represent the problem behind the decision to prescribe RAAS inhibitors: an attempt to balance the risk of developing hyperkalemia with benefits in relation to cardiovascular morbidity and mortality. These decisions are further complicated by the fact that patients with CKD with concomitant DM and / or HF who are most likely to experience the greatest benefit from these drugs are the same patients who have the highest risk of developing hyperkalemia [5,7].

\section{Management of Hyperkalimia}

There are differences between acute and chronic treatment, and how important this difference is in the treatment of hyperkalemia. Acute hyperkalemia is usually an emergency clinical situation that requires immediate intervention, such as heart monitoring, taking potassium-lowering drugs, or emergency dialysis. Typically, potassium levels $>5.5 \mathrm{mmol} / \mathrm{L}$ are considered clinically significant. The main goals for the treatment of severe, acute hyperkalemia are to stabilize the potential of the heart membrane and prevent cardiac arrhythmias, induce serum potassium displacement from the extracellular to intracellular space, and reduce the total serum potassium level [6].

Treatment options for the treatment of acute hyperkalemia can be further divided into the following categories: (a) those with an action within a few minutes that are more suitable for emergency care, and (b) those with an onset of action of several hours to provide therapeutic exposure and is suitable for intermediate or subacute care. Treatments include nebulized or inhaled beta-2 receptor agonists (eg, albuterol, salbutamol); intravenous (iv) insulin and glucose, which stimulate intracellular absorption of potassium; and IV gluconate calcium salt to stabilize the membrane. Kayexalate sodium bicarbonate, loop diuretics, dialysis and potassium-binding resins, and Kayexalate sodium polystyrenesulfonate (SPS) played a role in subacute hyperkalemia therapy as an adjunctive therapy after the initiation of emergency treatment [10]. In conditions of 
chronic treatment of hyperkalemia, it is important to identify any causes underlying the episode of hyperkalemia and to manage these factors / causes on an ongoing basis. Dietary potassium restriction and oral administration of sodium bicarbonate and loop diuretics can be used. SPS will sometimes be prescribed in chronic conditions, but due to poor taste, deep diarrhea, its associated side effects and salt loading, patients may not be committed to treatment. Patients with ESRD may use longer dialysis sessions, lower potassium levels in the dialysate, and restrict diet. Chronic hyperkalemia management is mainly based on RAASi therapy downtritation or withdrawal according to current available guidelines and potassium binders.

\section{RAASi Therapy Modulation}

Evidence-based medicine and current clinical practice suggest that inhibition of RAAS is a cornerstone for both cardioprotection in patients with heart failure and renoprotection in patients with diabetes (especially with concomitant hypertension). However, inhibition of RAAS may be difficult in patients with CKD due to the risk of progression to end-stage renal disease and / or the development of hyperkalemia.

The disadvantage of reducing or discontinuing RAASi therapy is that high-risk patients (both cardiovascular and diabetic) miss the best treatment option available, and this is closely related to the increasing incidence of serious cardiovascular events and mortality. Hypekalemia can be avoided by adding alkali to correct metabolic acidosis, prescribing a diet low in potassium and encouraging diuretic therapy, paying attention to subsequent hypovolemia and potential deterioration of major kidney diseases.

\section{Sodium Polystyrene Sulfonate}

Sodium polystyrenesulfonate (SPS) was, and still is, primary care for controlling potassium levels in patients with CKD and HF, but only 1 randomized controlled trial was conducted. As noted in everyday clinical practice, the most common side effects were nausea, vomiting, and constipation. SPS is commonly used in hospitals and less often on an outpatient basis due to low tolerance, since in 2009 the US Food and Drug Administration issued a warning due to reported cases of colon necrosis, bleeding, ischemic colitis and perforation associated with SPS therapy. Based on clinical reports, SPS therapy is not suitable for the treatment of chronic hyperkalemia, and new potassium binders are needed.

\section{The Role of Patiromer}

Until recently, treatment options for patients with chronic hyperkalemia were limited to non-pharmacological interventions (e.g., low potassium intake with food), loop diuretics with or without sodium bicarbonate and / or dose adjustment or discontinuation of therapy with RAAS inhibitors. Some doctors even gave fludrocortisone. These limited options are not optimal for most patients. Thus, there is an unmet need for a safe, effective, and well-tolerated chronic strategy to combat hyperkalemia. Before the onset of the patiromer, doctors usually considered the following management strategies: a) If the diet alone could not control potassium, the doses of diuretics were increased and bicarbonate replacement was considered; however, these treatments ultimately lose their effectiveness as patients lose their kidney function.

b) When the dose reduction of the RAAS inhibitor did not achieve the desired results, therapy with the RAAS inhibitor was eventually discontinued.

c) Some doctors even gave fludrocortisone with a risk of developing hypertension and vascular injuries.

On October 21, 2015, the patiromer received FDA approval for the treatment of hyperkalemia. Patiromer is a polymer that exchanges calcium for potassium. It has been shown to reduce and maintain serum potassium levels in patients with CKD who receive RAAS inhibitors. This medication is not indicated to prevent hyperkalemia. In addition, due to the delay in the onset of action, the patiromer is not intended for use as emergency therapy for lifethreatening hyperkalemia. The patiromer has the ability to bind to orally administered drugs, which may reduce their absorption and effectiveness. As a result, it is recommended that other oral medications be administered at least 3 hours before or 3 hours after the pyromere [11]. The two main clinical trials that contributed to the approval of the patiromer were OPAL-HK and AMETHYST-DN. A multinational, single-blind, 2-phase OPAL-HK study evaluated the efficacy of the patiromer in patients with CKD treated with RAAS inhibitors. At the first stage, all participants received an initial dose of patiromer in the amount of $8.4 \mathrm{~g}$ or $16.8 \mathrm{~g}$ per day in 2 divided doses, depending on the severity of hyperkalemia, at the beginning of the study for 4 weeks [12]. The study results showed an average decrease in serum potassium concentration of $1.01 \mathrm{mmol} / \mathrm{L}$, and in $76 \%$ of patients, serum potassium levels were within the target range of 3.8 to $\leq 5.1 \mathrm{mmol} / \mathrm{L}$ at the end of 4 weeks. In phase 2, the 8-week randomized withdrawal phase investigated the effects of continued patiromer compared to the transition to placebo. The results of stage 2 demonstrated that there were no changes in potassium levels in participants who continued the patiromer; however, patients who switched to placebo showed an average increase in serum potassium level of $0.72 \mathrm{mmol} / \mathrm{L}$ during the first 4 weeks of the phase. These results demonstrate the need for continuous treatment in this patient population and confirm the benefit of chronic use of the patiromere to maintain safe potassium levels. The 52-week, multicenter, open-label randomized trial AMETHYST-DN evaluated the safety and efficacy of the patiromer in hypertensive patients with diabetes or CKD [13]. Patients were stratified according to baseline serum potassium levels and received 1 out of 3 randomized starting doses ranging from $8.4 \mathrm{~g}$ to $33.6 \mathrm{~g}$ per day given in 2 divided doses. Doses were titrated to achieve and maintain serum potassium levels of $\leq 5.0 \mathrm{mmol} / \mathrm{L}$. The observed decrease in serum potassium levels ranged from $0.35 \mathrm{mmol} / \mathrm{L}$ for patients with the lowest dose to $0.92 \mathrm{mmol} / \mathrm{L}$ for patients with the highest dose. There was no evidence of loss of effectiveness within 1 year of treatment. The drug was also well tolerated with a small dropout rate. 
The third study, PEARL-HF, was a double-blind, randomized, placebo-controlled study that was designed to evaluate the safety and efficacy of the patiromer for 4 weeks in patients with chronic heart failure who were candidates for spironolactone and had serum potassium. the level is from 4.3 to $5.1 \mathrm{mmol} / \mathrm{L}$ [14]. Registered patients should also have had a history of documented hyperkalemia leading to cancellation of the aldosterone antagonist, ACE inhibitor, ARB, or beta-blocker or CKD with an estimated glomerular filtration rate $<60 \mathrm{ml} / \mathrm{min}$. An average decrease in serum potassium levels of $0.22 \mathrm{mmol} / \mathrm{L}$ from the baseline was observed in the group of patiromer compared to an average increase of $0.23 \mathrm{mmol} / \mathrm{L}$ from the baseline in the placebo group. With the simultaneous administration of spironolactone, patient treatment prevented an increase in potassium levels. The group indicated that, based on their clinical experience, they see the patiromer as a viable treatment option for patients with hyperkalemia, especially in chronic conditions, and that SPS and the patiromer can be used as adjunctive therapy after the start of emergency treatment. However, more evidence is needed to confirm their use in emergency situations. The group also stated that the advantage of the patiromer over SPS is its more favorable side effect profile, odorless formulation, and the use of calcium instead of sodium as an exchange ion, which can improve patient adherence. Based on $2-3 \%$ of cases of hyperkalemia in the general population, from 6.4 to 9.6 million people in the United States may qualify for treatment with a patiromer [15]. At present, many doctors are not familiar with the availability and administration of pyromeres, and the commission suggested that an educational campaign initiated by the payer, manufacturer, or provider of medical services may be useful to bridge this educational gap. Commissioners indicated that the educational campaign should target nephrologists, internal medicine doctors, family medicine, and emergency doctors, as they are primary or first-line providers and treat most of these patients. Educational campaigns should also be aimed at cardiologists and doctors who work in conditions of emergent departments, since it is in these conditions that most patients with hyperkalemia are treated, and then they are referred to a primary health care service or to a specialist. The expert group believed that the clinical level of use of the patiromer would be influenced by the existing level of comfort of doctors regarding other methods of treatment, the indecision of using new products until an adequate education or experience with new drugs on the market, as well as the potential for useful results among patients in their own practice.

\section{Conclusion}

Hyperkalemia is a serious problem in patients with CKD, HF and DM, especially in the treatment of RAASi. Modern management of hyperkalemia does not provide complete control of potassium levels over a long period of treatment. Discontinuation of RAASi therapy limits patients in the treatment of their underlying diseases, and patiomers are significant advances in the treatment of hyperkalemia. Patiromer has increased potassium specificity along with an improved safety profile compared to SPS. Based on current evidence, a patiromer seems to be the preferred treatment for hyperkalemia.

\section{Acknowledgement}

None.

\section{Conflict of Interest}

No conflict of interest.

\section{References}

1. Hollander-Rodriguez JC, Calvert JF Jr (2006) Hyperkalemia. Am Fam Physician 73(2): 283-290.

2. An JN, Lee JP, Jeon HJ, Kim DH, Oh YK, et al. (2012) Severe hyperkalemia requiring hospitaliza- tion: predictors of mortality. Crit Care 16(6): R225.

3. Epstein M, Alvarez PJ, Reaven NL, Funk SE, McGaughey KJ, et al. (2016) Evaluation of clinical outcomes and costs based on prescribed dose level of renin-angiotensin-aldosterone system inhibitors. Am J Manag Care 22(11 Suppl): S311-S324.

4. Luo J, Brunelli SM, Jensen DE, Yang A (2016) Association between serum potassium and outcomes in patients with reduced kidney function. Clin J Am Soc Nephrol 11(1): 90-100.

5. Uribarri J, Oh MS, Carroll HJ (1990) Hyperkalemia in diabetes mellitus. J Diabet Complications 4(1): 3-7.

6. Jain N, Kotla S, Little BB, Weideman RA, Brilakis ES, et al. (2012) Predictors of hyperkalemia and death in patients with cardiac and renal disease. Am J Cardiol 109(10): 1510-1513.

7. Reaven NR, Funk SE, Bakris GL (2014) The economics of renoprotective therapy in advanced diabetic kidney disease. Am J Pharm Benefits 6(6): e169-e176.

8. Lehnhardt A, Kemper MJ (2011) Pathogenesis, diagnosis and management of hyperkalemia. Pediatr Nephrol 26(3): 377-384.

9. Raebel MA, Ross C, Xu S, Roblin DW, Cheetham C, et al. (2010) Diabetes and drug-associated hyperkalemia: effect of potassium monitoring. J Gen Intern Med 25(4): 326-333.

10. Weir MR, Bakris GL, Pitt B (2015) New agents for hyperkalemia. N Engl J Med 372(16): 1570-1571.

11. Sterns R, Grieff M, Bernstein P (2016) Treatment of hyperkalemia: something old, something new. Kidney Int 89(3): 546-554.

12. Weir MR, Bakris GL, Bushinsky DA, Mayo MR, Garza D, et al. (2015) Patiromer in patients with kidney disease and hyperkalemia receiving RAAS inhibitors. N Engl J Med 372(3): 211-221.

13. Bakris GL, Pitt B, Weir MR, Freeman MW, Mayo MR, et al. (2015) Effect of patiromer on serum potas- sium levels in patients with hyperkalemia and diabetic kidney disease: the AMETHYST-DN randomized clinical trial. JAMA 314(2): 151-161.

14. Pitt B, Anker SD, Bushinsky DA, Kitzman DW, Zannad F, et al. (2011) Evaluation of the efficacy and safety of RLY5016, a polymeric potassium binder, in a double-blind, placebo-controlled study in patients with chronic heart failure (the PEARL-HF) trial. Eur Heart J 32(7): 820-828.

15. Chainman M, Dixit D, Bridgeman MB (2016) Potassium-binding agents for the clinical management of hyperkalemia. PT 41(1): 43-50. 